1) eine Flasche mit Jösung I

2) eine Flasche mit Lösung II

3) Mischgefäß mit 2 Marken zum Abmessen gleicher Raumteile

4) Papprolle mit Fließpapierstreifen 5) Farbmuster (Papierstreifen in Reagenzglas)

6) sechs leere dickwandige Reagenzgläser mit Korken.

Die Gebrauchsanweisung für die "Gasrestprobe" lautet:

a) Man füllt das Mischgefäß bis zur Marke 1 mit Lösung I, sodann bis zur Marke 2 mit Losung II und schüttelt nach Aufsetzen des Glasstopfens durch.

b) Man befeuchtet durch Eintauchen in das Mischgefä $\beta$ die unteren Hälften von sechs Fließpapierstreifen mit der Mischung, steckt jeden Streifen in ein besonderes Glasrohr und stopft zu.

c) Die Prüfung eines durchgasten Raumes auf Blausäure darf nicht sofort nach der Durchlüftung vorgenommen werden, vielmehr miissen die Fenster und Türen des Raumes mindestens 1 Stunde vor der Prüfung geschlossen gewesen sein.

Man betritt mit angelegtem Schutzgerät den auf Blausäure zu prüfenden Raum mit den sechs in Glasrohre eingeschlossenen, nach a) und b) frisch bereiteten feuchten Reagenzpapierstreifen. An einer von Fenster und Türen möglichst entfernten Stelle entnimm man einen Streifen aus dem Glasrohr und beginnt gleichzeitig langsam von 21 bis 27 zu zählen. Färbt sich während dieser Zeit (7 Sek.) das Reagenzpapier nicht deutlich blau (wie Farbmuster), so besteht in der geprüften Stelle keine Blausäuregefahr mehr. Diese Prüfung ist an verschiedenen möglichst weit auseinanderliegenden Stellen und in verschiedener Höhe über dem Fußßboden noch zweimal zu wiederholen. Bei der Prüfung sind besonders die sogenannten Gefahrstellen ${ }^{16}$ ) $\mathrm{zu}$ bevorzugen. Zeigt sich an keiner Stelle des Raumes eine Blaufärbung der Reagenzpapierstreifen, so kann der Raum unbedenklich dauernd benutzt werden, andernfalls ist die ganze Prüfung zu wiederholen, nachdem nochmals längere Zeit gelüftet worden ist, und die Fenster und Türen im Anschluß daran mindestens 1. Stunde geschlossen gewesen sind. -

Die unvermischten Lösungen I und II sind, wenn sie gut verschlossen und im Dunkeln aufbewahrt werden, haltbar. - Das gemischte Reagens ist höchstens 2 Wochen brauchbar. Ein dunkler, flockiger Bodensat $\%$ in der Mischung zeigt an, daß sie unbrauchbar geworden ist.

Anhang.

Bemerkungen über die Darstellung gasförmiger Blausäure.

Das einfachste Verfahren, gasförmige Blausäure zu entwickeln ist die Einwirkung von mäßig verdünnter Schwefelsäure auf Natriumcyanid in offenen Bottichen. ${ }^{17}$ (Bottichverfahren.) 60 grädige Schwefelsäure wird eben vor der Vergasung mit Wasser vermischt, und das Cyanid sofort in die noch heiße Mischung eingetragen. Wenn bei der Vergasung wie gewöhnlich ein Gehalt von 1 Vol $\%$ HCN $\left(12 \mathrm{~g} \mathrm{~m}^{3}\right)$ in der Luft verlangt wird, so werden für $100 \mathrm{cbm}$ Raum stat der theoretiseh elforderichen Menge von $2,2 \mathrm{~kg}$ NaCN meisten $2,5-2,75 \mathrm{~kg}$ zersetzt, um den Verunreinigungen des technischen Cyanids Ind unvermeidlichen Verlusten Rechnung zu tragen. Für die Zersetzung von $1 \mathrm{~kg}$ reinem $\mathrm{NaCN}$ ist gerade $1,0 \mathrm{~kg} \mathrm{H} \mathrm{H}_{2} \mathrm{SO}_{4}\left(100 \% \%_{0} \mathrm{ig}\right)$ oder 1,28 kg $(0,75 \mathrm{l}) \mathrm{H}_{2} \mathrm{SO}_{4}$ von $60^{\circ} \mathrm{Bé}\left(78 \mathrm{Gew}^{0}, \mathrm{H}_{2} \mathrm{SO}_{4} ; \quad \mathrm{d}=1,71\right)$ notwendig. Die technischen Vorschriften schwanken zwischen 1,5 und $2,6 \mathrm{~kg}$ Sïure von 60 $0^{\circ}$ Bé. Die Wassermengen werden so angegeben, daß nach der Mischung eine Säure von 30-40 Gew ${ }^{0}$ $\mathrm{H}_{2} \mathrm{SO}_{4}$ entsteht.

Zahlreiche quantitative Versuche, die von uns zum Teil in Gemeinschaft mit Herm Dr. Wir th ausgeführt wurden, ergaben, daß auf $1 \mathrm{~kg} \mathrm{NaCN}$ nicht mehr als $1,6 \mathrm{~kg}(0,94 \mathrm{l}) 60^{\circ}$ iger Säure erfordert werden. Die Schwefelsäure ist mit dem gleichen Gewicht Wasser za verdünnen. Nimmt man mehr Wasser, so hinterbleibt mehr Blausïure in der Flüssigkeit; nimmt man zu wenig Wasser, so kristallisier beim Erkalten leicht ein Teil des Sulfats aus. Für die Vergasung von je $100 \mathrm{cbm}$ Raum mit $1 \mathrm{Vol}^{0}:$ Blausäure ist demnach folgende Vorschrift zweckmäßig:

In $4,4 \mathrm{l}$ Wasser, das womöglich auf $50-60^{\circ}$ vorzuwärmen ist werden $2,6 \mathrm{I} \mathrm{H}_{2} \mathrm{SO}_{4}$ von $60^{\circ}$ Bế gegossen und in die heiße Mischung möglichst bald $2,75 \mathrm{~kg} \mathrm{NaCN}$ eingeworfen.

"Geschmolzenes" Cyanid hinterläßt leicht einen Rückstand, besonders wenn die Stücke in Papierumhüllung eingeworfen werden. Empfehlenswerter sind die in den Handel gebrachten etwa $450 \mathrm{~g}$ schweren Briketts. Sie werden (ohne Hülle) sehr rasch und vollständig zersetzt. Um ein Überlaufen der stark schäumenden Lösung zll verhindern, sollte nach unseren Erfahrungen die Füllhöhe weniger als ein Viertel der Bottichhöhe betragen. Bei der Reaktion verdampfen erhebliche Wassermengen: bei der Vergasung von drei Räumen mit je $4,5 \mathrm{~kg}$ NaCN-Briketts wurden beispielsweise $0,5-0,7 \mathrm{~kg}$ verdampftes Wasser auf $1 \mathrm{~kg}$ zersetztes Cyanid gefunden.

Es sei endlich erwähnt, daf mit Natriumbisulfat gleichgute Gasausbeiten erhalten werden können wie mit Schwefelsăiure, wenn

16) „Totc Winkel, durch Möbel verstellte Ecken, Nischen, Schı̈̈nke, Lüftungs- und Heizungsschächte, Kachelöfen, poröse Wände, Wandfüllungen, feuchte Ecken usw: "

17) Vgl z. B. Bail, I. e. und Skramlik, l. c. auf $1 \mathrm{~kg}$ NaCN eine kochend heiße Lösung von $4,8 \mathrm{~kg}$ Bisulfat in $9,6 \mathrm{~kg}$ Wasser verwendet wird. Unter diesen Bedingungen bleibt auch nach dem Erkalten alles in Lossung.

Die nach der angegebenen Vorschrift erhaltenen Ausbeuten an gasförmiger Blausäure lagen zwischen 80 und $90 \%$

Greifswald, d. 21. Nov. 1920.

\section{Über eine kolorimetrische Arsenbestimmung im Harn und Blut von mit Salvarsan usw. behandelten Patienten.}

(Mitteilung aus dem staatlichen Hygienischen Institut Hamburg, Direktor Prof. I)r. Von Dr. KuRT SCHEFFleR.

Die moderne Salvarsan-Therapie und die sich aus ihr ergebenden Fragen über die schädlichen Wirkungen des Arsens auf den menschlichen Körper nehmen in der Fachliteratur der letzten Jahre einen recht breiten Raum ein. Es fehlt auch nicht an geeigneten Vorschlägen und Methoden zur quantitativen Bestimmung des Salvarsans oder des Arsens im Blute oder den Sekreten von mit diesen Präparaten behandelten Patienten.

So sind besonders interessant die Arbeiten von I. Abelin ${ }^{1}$ ), die auf eine Bestimmung des Salversans als solches hinausläuft, ferner die von M. Vinograd*), C. R. Sanger und O. F. Black ${ }^{3}$, welche beide letzten Endes den Marshapparat benutzen, von G. Ot to Gaebel4), von Bohrisch und Kürschner ${ }^{5}$ ), sowie A. Heiduschka und Th. Biéchy ${ }^{6}$, die eine jodometrische Methode anwenden, von W. Ko sia n T, der Arsen mit bromsaurem Kali titriert, von Merk u ri ew ${ }^{8}$ ), der eine Methode empfiehlt, die auf die Bildung von HgS hinausläuft und die Vergleichung der Intensität eines Farbenfleckens dieser Verbindung auf Papier zum Ziele hat, von Pa uI Duret $t^{(3)}$, der den im Marshapparat entwickelten Arsenwasserst off in Silbernitratlossung auffängt und einer Anzahl anderer Autoren, deren Erwähnung an dieser Stelle zu weit führen würde.

Es ist nun nicht meine Absicht, diese Methoden zu kritisieren und ihre Brauchbarkeit nachzuprüfen, sondern es kommt mir heute nur darauf an, eine hier bei uns im Institut auf Anregung von Herrn Prof. Dr. Noll ausgeführte Methode der Bestimmung kleiner Arsenmengen in physiologischen Flüssigkeiten auf kolorimetrischem Wege z.u beschreiben und zur Verwendung zu empfehlen.

Die vorliegende Arbeit wurde bereits vor dem Kriege im hiesigen Institut von Dr. F. Gothe und mir begonnen. Da ich bereits Anfang August 1914, Dr. Gothe im Laufe des Jahres 1915 zum Heeresdienst einberufen wurde, so mußte die Veroffentlichung dieser Arbeit verschoben werden, zumal noch eine Anzahl Nachprüfungen derselben nötig waren. Nach 5jähriger Abwesenheit kehrte ich im November 1919 nach Hamburg zurilck und konnte dann erst wieder meine Untersuchungen in diesem Gebiet aufnehmen. Dr. Gothe ist leider seit der Cambraischlacht des Jahres 1917 vermißt, und ich sehe mich daher genötigt, auch seine Untersuchungsergebnisse mit unter meinem Aamen zu veröffentlichen.

Die von uns zwecks Festlegung einer schnellen Methode zur Bestimmung von Arsen im Harn, Blut usw. angestellten Versuche haben ergeben, daß die Methode von Bettend orf dazu geeignet ist. Diese beruht bekanntlich darauf, daß das Arsen mit Hilfe von Zinnchlorür aus seinen Verbindungen metallisch abgeschieden wird. Fällt der Arsengehalt einer Lösung unter eine gewisse Grenze, so ermöglicht es die dabei eintretende Braunfärbung verschiedener Intensität, die in den Flüssigkeiten enthaltenen Arsenmengen nach den üblichen kolorimetrischen Methoden $z u$ bestimmen.

Ich möchte an dieser Stelle gleich erwähnen, daß in den nach dieser Methode untersuchten Flïssigkeiten der Arsengehalt auch naoh der Marsh schen Methode festgestellt wurde, die bekanntlich darauf beruht, daß das Arsen als Spiegel oder Flecken abgeschieden wird. Die Menge des Arsens wird dabei entweder gewichtsanalytisch oder

1) I. Abelin, Über eine neue Methode, das Salvarsan nachzuweisen. (Vorl. Mitt.) Münch. med. Wochenschr. $1911, \mathrm{I} / 1002$.

2) Mariam Vinograd, Zur Bestimmung von Arsen in organischen Subtanzen. J. Am. Chem. Soc. 36, 1548.

3) C. R.S a nger und O. F. B lack, Bestimmung von Arsen im Urin. Z. anorg. Chem. 56, 153

t) G. Otto Ga ebel, Das Salvarsan beim gerichtlichen Arsennachweis. Ar. . Pharmacie 1911 (Bd. 249).

5) P. Bohrisch und F. Kürschner, Zur quantitativen Bestimmung des Arsens in organ. Substanzen mit bes. Berïcksichtigung organ. Arsenverbindungen. Pharm. Zentralh. 52, $1365 \mathrm{ff}$, $1397 \mathrm{ff}$.

(7) A. Heiduschka und Th. Biéchy, Bestimmung des Arsens im Harn nach Anwendung von Salvarsan. Apothekerztg. 26, 146.

7) Wilbelm Kosian, Eine Methode der quantitativen Bestimmung von Arsen im Harn. Pharm. Post 48, 321.

8) W. A. Merkuriew, Arsennachweis im Harn nach del Anwendung von Salvarsan. Wiener klin. Wochenschr. 25, 588 .

9) Paul Duret, Nachweis, Bestimmung und Ausscheidung dos Arsens in Urin. C. r. Soc. de Biol. 81, 736 . 
durch Vergleichsspiegel ermittelt. Beide Methoden wichen aber recht häufig ganz bedeutend voneinander $a b$, was darauf zurückzuführen ist, daß die Spiegel bei gleich großen Arsenmengen oft sehr verschiedenen Flächenraum einnehmen und sich daher zu Vergleichen nicht eignen. Auch die gewichtsanalytische Bestimmung so kleiner Arsenmengen, wie sie bei den in Frage stehenden Untersuchungen vorkommen, begegnet größeren Schwierigkeiten, und wir haben daher von der $\mathrm{Me}-$ thode nach Marsh in der Folge ganz Abstand genommen.

Als Voraussetzung für die Brauchbarkeit einer kolorimetrischen Methode mußte vor allen Dingen darauf gesehen werden, die betreffenden Flüssigkeiten in völlig farblosem Zustande zu erhalten, und dies ist uns nach einer Reihe von Versuchen auch restlos gelungen, so daß auch dieser Umstand für eine erfolgreiche Anwendung der Methode nach Bettend orf spricht.

Zur Entfärbung und Aufschließung der organischen Substanzen wurden verwendet: Blutkohle, Kaliumpermanganat und eine Mischung von Bleiessig und Baryumhydroxyd, ohne daß es durch diese Reagentien gelungen wäre, genügend klare und farblose Flüssigkeiten zu erhalten, die sich zur Verwendung unserer Bestimmung mit $B$ ettend orf Reagens eigneten. Endlich schlugen wir den schon von FrenkelHeiden und E. Navassart ${ }^{10}$ ) vorgeschlagenen Weg der Aufschließung mit Schwefelsalpetersäure $(1+4)$ ein.

Diese Mischung erwies sich zur völligen farblosen Aufschließung des Harns als durchaus geeignet und wurde von uns in der Folge stets ingewendet. Die Methodik dieser Aufschließung, die wir nach einigen Versuchen für unsere $Z$ wecke als die geeignetste feststellten, ist folgende:

$100 \mathrm{ccm}$ Harn, bei zu erwartenden größeren Arsenmengen (erste Tage nach der Salvarsanbehandlung!) entsprechend weniger, werden in einer Porzellanschale bis auf etwa $1 / 5$ des Volumens eingedampft, dann $25 \mathrm{ccm}$ des Säuregemisches zugegeben und weiter bis zur ungefähren Schwefelsäurekonsistenz eingedickt. Das noch gelbliche Gemisch wird unter Nachspülen mit dreimal etwa $5 \mathrm{ccm}$ Säuregemisch in einen Kjeldahlkolben von $100 \mathrm{eem}$ übergeführt und auf dem Drahtnetz weiter erhitzt. Es findet ein im allgemeinen unerhebliches Stoßen statt, dann wird die Flüssigkeit allmählich tief dunkelbraun bis schwarz. In diesem Zustande läßt man dieselbe etwa 15 Minuten ruhig sieden, dann fügt man zu dem noch kochendheißen Gemisch etwa $2 \mathrm{ccm}$ des Säuregemisches hinzu. Unter starkem Aufschäumen und Entwicklung roter nitroser Dämpfe wird die Flüssigkeit alsbald hellgelb und nach kurzer Zeit ganz farblos, was man beschleunigen kann, indem man nach einiger Zeit (etwa 5 Minuten) noch einige Tropfen des Säuregemisches hinzufügt. Die Aufschließung ist nun praktisch beendet. Man kocht nun noch etwa 20 Minuten weiter, bis auch die letzten Spuren Salpetersäure, die event. auf das Zinnchlorür im Bettend orf schen Reagens oxydierend einwirken könnten, verschwunden sind. Eine Probe so aufgeschlossenen Harn-Säuregemisches gab init Brucin nur eine geringe Gelbfärbung.

Auf die soeben beschriebene Weise erhält man 5-7 ccm eine farblosen, sirupdicken Flüssigkeit. Es gelang nun nicht, auf Zusatz der 3-5 fachen Menge von Bettend orfs Reagens direkt zu dieser konzentrierten Flüssigkeit das Arsen kolorimetrisch zu bestimmen. Es bildeten sich Niederschlïge, welche jedenfalls das fein verteilte reduzierte Arsen mitrissen, so daß es sich der Beobachtung auf kolorimetrischem Woge entzog. Verdünnt man dagegen die Flüssigkeit mit Wasser auf $25 \mathrm{ccm}$, fügt nun $75 \mathrm{ccm}$ Bettend orfs Reagens hinzu, kocht die Iösung kurz auf und läßt eine halbe Stunde stehen, so kann man dieselbe im Hehnerschen Zylinder direkt mit Vergleichsflüssigkeiten einstellen, und es gelang auf diese Art, das Arsen schnell und sicher nachzuweisen.

Es sei an dieser Stelle bemerkt, daß wir bei unseren Versuchen die von Winkler ${ }^{11}$ ) vorgeschlagene Herstellung von Bettendorfs Reagens in der Weise modifizierten, daß wir zur Lobsung des Stanno-

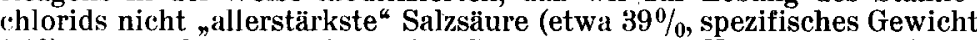
1,19) verwendeten, sondern eine Säure geringerer Konzentration (etwa 25"\% ig, spezifisches Gewicht 1,124). Die Dämpfe der stark rauchenden Säure der hohen Konzentration beeinflußsten die Beobachtung der Farbentöne in den zu vergleichenden Flüssigkeiten in höchst störendem Maße, und wir erhielten mit unserem Reagens, wie weiter unten gezeigt werden wird, durchaus einwandfreie Resultate.

Da im übrigen das Bettend orf sche Reagens beim Aufbewahren durch Übergang des Zinnchlorïrs in Chlorid unwirksam wird, können wir nach unseren Erfahrungen zu seiner Konservierung das bekannte Einstellen einer Zinnstange in die Flüssigkeit empfehlen. Hierdurch wird das Reagens wieder wirksam; vom abgeschiedenen Zinn filtriert man durch Watte oder Asbest ab.

Hierbei ist zu bemerken, daß Harn ohne Arsenzusatz auf diese Art aufgeschlossen und verdünnt ebenfalls eine Färbung, nämlich einen Stich ins Grüngelbliche gibt. Um diese Färbung möglichst auszuschalten, stellte ich mir eine sogen. Harnschwefelsäure dar, die ich statt gewöhnlicher Schwefelsäure zur Vergleichsflüssigkeit zugab. Es wurden zu diesem Zweck immer $100 \mathrm{ccm}$ Harn mit $25 \mathrm{ccm}$ Schwefelsalpetersäure wie oben aufgeschlossen und die so erhaltene Flüssigkeit als "Harnschwefelsäure" gesammelt. Von dieser Harnschwefel-

10) Frenkel-Heiden und E. Navassart, Über die Elimination des Salvarsans aus dem menschl. Körper. Berl. klin. Wochenschr. 1911, Nr. 30.

11) L. W. Winkler, Über den Nachweis des Arsens mit Bettendorfschem Reagens. Z. angew. Chem. 26, Nr. 19 säure wird zur Vergleichslösung genau dieselbe Menge zugegeben wie die aufgeschlossene Flüssigkeitsmenge des Untersuchungsharnes beträgt, da andernfalls die Resultate ungenau werden.

Ubber die Genauigkeitsgrenze der Methode wäre zu bemerken, daß Mengen von 0,05 und $0,04 \mathrm{mg}$ As in $100 \mathrm{ccm}$ Harn (also 0,5 resp. $0,4 \mathrm{mg}$ im Liter) noch quantitativ nachgewiesen werden können, während unterhalb dieser Grenze die Färbung so unbestimmt wird, daß sie sich zum genauen quantitativen Nachweis nicht mehr gut eignet. $0,03 \mathrm{mg} \mathrm{As}$ in $100 \mathrm{ccm}$ Harn ist eben noch festzustellen, darunter muß. man sich mit einem Identitätsnachweis des Arsens begnügen. Als solcher erwies sich die Gutzeitsche Probe als durchaus geeignet. Das aufgeschlossene Harngemisch wird entweder im Aufschließßungsgefäß selbst oder in einem kleinen Erlenmeyer-Kölbchen mit etwa der 5 fachen Wassermenge verdünnt und ein Stückchen arsenfreies Zink, von dessen Reinbeit man sich durch einen blinden Versuch überzeugen muß, hineingetan. Es setzt eine ziemlich lebhafte Wasserstoffentwicklung ein. Man verschließt nun, um alle Feuchtigkeit abzusperren, das Gefäß lose mit einem Wattestopfen und deckt auf die Öffnung ein Stückchen quantitatives Filtrierpapier, das mit einem Tropfen konzentrierter Silbernitratlösung $(2+1)$ befeuchtet wird.

Bei Anwesenheit von Arsen färbt sich die mit der Silberlösung befeuchtete Stelle des Papiers kanariengelb, läßt man nun auf dieselbe einen Tropfen Wasser fallen, so wird der gelbe Fleck schwarz nach den bekannten Gleichungen:

$$
\begin{gathered}
2 \mathrm{As} \mathrm{H}_{3}+12 \mathrm{AgNO}_{3}=2\left(\mathrm{As} \mathrm{Ag}_{3}, 3 \mathrm{Ag} \mathrm{NO}\right)-6 \mathrm{H} \mathrm{NO}_{3} \\
2\left(\mathrm{As} \mathrm{Ag}_{3}, 3 \mathrm{AgNO}_{3}\right)+3 \mathrm{H}_{2} \mathrm{O}=\mathrm{As}_{2} \mathrm{O}_{3}+12 \mathrm{Ag}+6 \mathrm{H} \mathrm{NO}_{3} .
\end{gathered}
$$

Es ist streng darauf zu achten, daß jede Spur von Feuchtigkeit ferngehalten wird, da man sonst keinen rein kanariengelben Fleck erhält, sondern unbestimmte Mischfarben, die zu Täuschungen Anlaß geben können.

Auf diese Art gelingt es, noch niedrigere Arsenmengen als $0,03 \mathrm{mg}$ in $100 \mathrm{ccm}$ Harn bequem zu identifizieren. Meine Versuche in dieser Richtung mit Mengen von $0,02 \mathrm{resp} .0,01 \mathrm{mg}$, ja sogar $0,005 \mathrm{mg}$ As in $100 \mathrm{ccm}$ Harn gaben durchaus deutliche einwandfreie Reaktionen nach Gutzeit.

Fin Versuch mit normalem As-freien Harn gab nach Gutzeit zwar auch noch eine geringe Färbung, die ich aber nicht als kanariengelb bezeichnen konnte, und die gan $\mathrm{z}$ deutli $\mathrm{ch}$ von der mit $0,005 \mathrm{mg} \mathrm{As}$ $\mathrm{zu}$ unterscheiden ist.

In welcher Form das Arsen im Harn vorhanden ist, bleibt hierbei gleichgültig. ' Schon Ga eb el ${ }^{4}$ ) sagt, daß Salvarsan durch Salzsäure und Kaliumchlorat völlig mineralisiert wird. Ich bin wohl berechtigt, anzunehmen, daß dies durch das kräftige Säuregemisch Schwefelsalpetersäure $(1+4)$ ebenfalls in vollem Maße geschieht und habe bei der Bearbeitung des experimentellen Teiles der vorliegenden Arbeit meistens eine Lösung von arsenigsaurem Natrium als As-Zusatz benutzt, während Gothe, der die ersten Versuche gemeinschaftlich mit mir unternahm, sowohl Salvarsan- als auch Arsalytlösungen neben Lösúngen von arsenigsaurem Natrium als Arsenquelle benutzte und zugleich befriedigenden Resultaten gelangte.

Als Beleg hierfür mögen folgende Analysenergebnisse dienen:

\begin{tabular}{|c|c|c|c|}
\hline $\begin{array}{l}\text { Zugesetzte Menge } \\
\text { As in mg }\end{array}$ & $\begin{array}{l}\text { Wiedergefundene } \\
\text { Menge As in } \mathrm{mg}\end{array}$ & $\begin{array}{l}\text { Zugesetzte Menge } \\
\text { As in } \mathrm{mg}\end{array}$ & $\begin{array}{l}\text { Wiedergefundene } \\
\text { Menge As in mg }\end{array}$ \\
\hline & 6,0 & $0,45(A)$ & 0,43 \\
\hline 4,5 (S) & 4,5 & 0,20 (A) & 0,24 \\
\hline 2,1 (S) & 2,16 & 0,15 (A) & 0,15 \\
\hline $1,5 \quad$ (A) & 1,56 & $0,10(\mathrm{~A})$ & 0,10 \\
\hline 0,75 (S) & 0.75 & 0,05 (A) & 0,06 \\
\hline
\end{tabular}

I. Zusatz von As in Form von Salvarsan (S) resp. Arsalyt (A).

II. Zusatz von As in Form von arsenigsaurem Natrium.

\begin{tabular}{c|c|c|c|c|c}
\hline $\begin{array}{c}\text { Zugesetzt } \\
\text { mg As }\end{array}$ & $\begin{array}{c}\text { Wieder- } \\
\text { gefunden }\end{array}$ & $\begin{array}{c}\text { Zugesetzt } \\
\text { mg As }\end{array}$ & $\begin{array}{c}\text { Wieder- } \\
\text { gefunden }\end{array}$ & $\begin{array}{c}\text { Zugesetzt } \\
\text { mg As }\end{array}$ & $\begin{array}{c}\text { Wieder- } \\
\text { gefunden }\end{array}$ \\
\hline 0,90 & 0,70 & $\mathbf{0 , 2 0}$ & $\mathbf{0 , 2 0}$ & $\mathbf{0 , 0 6}$ & $\mathbf{0 , 0 5 5}$ \\
0,80 & 0,75 & 0,18 & $\mathbf{0 , 1 1}$ & $\mathbf{0 , 0 5}$ & $\mathbf{0 , 0 5}$ \\
0,75 & $\mathbf{0 , 7 1}$ & $\mathbf{0 , 1 6}$ & $\mathbf{0 , 1 5}$ & $\mathbf{0 , 0 4}$ & $\mathbf{0 , 0 5}$ \\
$\mathbf{0 , 6 0}$ & $\mathbf{0 , 6 3}$ & $\mathbf{0 , 1 4}$ & $\mathbf{0 , 1 0}$ & $\mathbf{6 , 0 4}$ & $\mathbf{0 , 0 4}$ \\
$\mathbf{0 , 5 0}$ & $\mathbf{0 , 4 2}$ & $\mathbf{0 , 1 0}$ & $\mathbf{0 , 0 9}$ & $\mathbf{0 , 0 3}$ & $\mathbf{0 , 0 2 5}$ \\
$\mathbf{0 , 5 0}$ & $\mathbf{0 , 4 5}$ & $\mathbf{0 , 0 8}$ & $\mathbf{0 , 0 7 5}$ & &
\end{tabular}

Werden Patienten mit Salvarsan, Arsalyt oder einem ähnlichen Arsenprïparat durch Injektion behandelt, so wird nach Beobachtungen von Abelin ${ }^{12}$ ) das Präparat hauptsächlich durch den Harn wieder ausgeschieden und nur zu einem kleinen Teil durch den Kot. Über den Beginn und die Dauer der Ausscheidung gehen die Meinungen verschiedener Autoren, wie Abelin in der soeben zitierten Abhandlung

12) 1. Abelin, Beginn und Dauer der Ausscheidung des Salvarsans durch den Urin nach intravenöser Injektion. Münch. med. Wochenschr. 1911, II $/ 1771$ 
sagt, etwas auseinander; er kommt jedoch zu dem Resultat, daß die Ausscheidung fast unmittelbar (5--10-15 Minuten) nach der Injektion beginnt, am ersten Tage nach der Injektion noch ziemlich bedeutend ist, dann aber allmählich abnimmt und nach längerer oder kürzerer Zeit gänzlich verschwindet. Seine Methode des Nachweises von Salvarsan im Harn als solches gestattet ihm nach seiner Angabe, diese Arsenausscheidung als Salvarsan zeitlich zu verfolgen. Auch unsere Methode erwies sich hierfür als durchaus geeignet, wie wir durch folgende Versuche feststellen konnten:

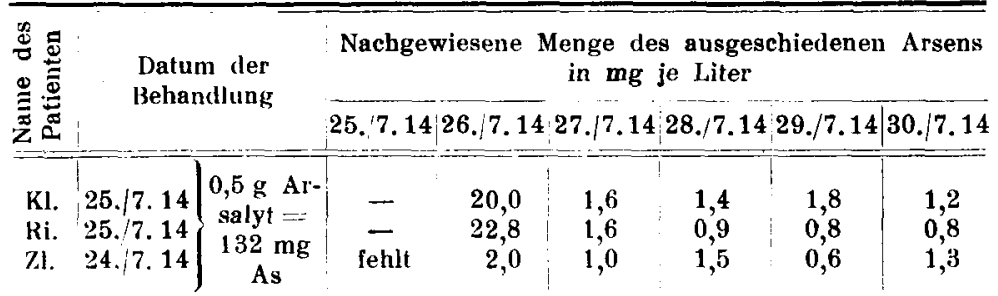

Unsere Methode gestattet, diese Untersuchungen nun beliebig lange fortzusetzen. Versagt schließlich die quantitative Bestimmung mittels der kolorimetrischen Methode, wie gezeigt, bei Mengen unter 0,03 mg, so gibt uns die qualitative Probe nach Gutzeit immerhin noch die Möglichkeit, die geringsten Spuren von Arsen in dem Untersuchungsmaterial $z$ identifizieren.

Das zur Verwendung kommende Volumen Harn richtet sich also nach dem 'Tage der Ausscheidung. Da am ersten Tage nach der Injektion die durch Harn ausgeschiedene Arsenmenge ziemlich erheblich ist, so verwendet man hier zweckmäßig nur etwa $10-20 \mathrm{ccm}$ und vergrößert an den folgenden Tagen entsprechend das zur Untersuchung verwendete Volumen bis auf $100 \mathrm{ccm}$, für die qualitative Probe event. aut 200 resp. $500 \mathrm{ccm}$.

Endlich möchte ich nicht verfehlen, darauf hinzuweisen, daß diese Methode voraussichtlich auch auf Nahrungsmittel und Gebrauchsgegenstände (Tapeten usw.) und auf Arsenbestimmungen in forensischen Fillen ibertragen werden kann.

[A. 229.]

\section{Zur Normung von Lufttrockenschränken.}

\author{
Von Dr. H. Gerber.
}

Aus der Werkstatt von Dr. Rob. Muencke.

Um einen Körper zu trocknen, führt man ihm entweder durch direktes Erhitzen Wärme zu oder, wo dies nicht angängig ist, bedient man sich der Luft als Vermittlerin. Alle im Laboratorium gebrauchten Trockenschränke sind Lufttrockenschränke, und zwar unterscheidet man solche, bei denen die Luft durch die Flamme erwärmt wird und solche, bei denen Wasser oder andere höher siedende Flüssigkeiten von möglichst konstantem Siedepunkt bis zum Siedepunkt erhitzt werden, und dann ihre Wärme an die Luft im Trockenschrank abgeben. Luft kann je nach ihrer Temperatur eine bestimmte Menge Wasserdampf aufnehmen, und man muß dafür Sorge tragen, daß die gesättigte Luft durch Ventilation abgeführt und durch neue trockene ersetzt wird. In einwandfreier Weise geschieht das in einem Trockensystem, das demjenigen der Großtechnik nachgebildet ist und von Buxton und Bebe besonders zur Trocknung von eiweißhaltigen Körpern im Laboratorium empfohlen wurde. Durch einen elektrischen Ventilator wird luft, die auf bestimmte Temperatur vorgewärmt worden ist, in ein System horizontaler Kammern geleitet, auf deren Boden sich in flachen Gefäßen die zu trocknende Substanz befindet. Man kann für gewöhnliche. Zwecke mit einfacheren Vorrichtungen auskommen, aber man muß dann für ausreichende Ventilation in erster Linie Sorge tragen. Sinngemäß darf man dann nicht etwa verlangen, daß im Innern eines Schrankes in den einzelnen Etagen die gleiche Temperatur herrscht, denn die eintretende vorgewärmte Luft wird einen Teil ihrer Wärme zur Bildung von Wasserdampf abgeben und sich abkühlen. Eine gleichmäßige Temperatur im Innern kann man hingegen von einem Brutapparat für bakteriologische $Z$ wecke verlangen, der wie ein Trockenschrank mit Wasserfüllung gebaut ist, seiner Bestimmung nach aber nicht unter diese Betrarhtungen fällt.

Die kleinen einfachwandigen Kästen erfüllen nur scblecht die an sie zu stellenden Ansprüche. Gegenstände, die sich auf dem unteren Einlageboden befinden, werden überhitzt, und eine Durchlüftung ist nur sehr unvollkommen, besonders wenn die eine der beiden Tuben durch ein Thermometer verschlossen ist. Die Tür müßte einen Schieber für die Luftzirkulation besitzen und die Tuben einen Aubendurchmesser von $20 \mathrm{~mm}$ haben. Zum Aufhängen des Trockenschrankes müßten immer Ốsen vorhanden sein. Die Füße dürtten nicht angenietet sein, sondern ein Vierfuß müßte nachträglich zu jedem Kasten passend geliefert werden kónnen. Als Material empfehle ich Aluminium und möchte dabei daran erinnern, daß vor Jahresfrist die Physikalisch technische Reichsanstalt eine Umfrage, wie sich dieses Material in Lahoratorien bewähre, veranstaltet hat; vielleicht dürfte das Resultat inzwischen vorliegen. Von den zehn Größen, in denen diese Kästen angefertigt wurden, genügt eine von $15 \mathrm{~cm}$ Höhe, $29 \mathrm{~cm}$ Breite, $15 \mathrm{~cm}$ Tiefe Einen Fortschritt in der Konstruktion bedeutet ein Aluminiumkasten ähnlicher Größe, der im I. Chemischen Institut in Berlin in Gebrauch ist, und ich schlage vor, diese eine Grőße beizubehalten. Dieser Kasten hat einen doppelten Boden, und die vorgewärmte Luft durchstreift den Innenraum.

Wesentlich besser sind die Kästen, bei denen die Heizgase an den Seitenwänden und der Rückwand in die Höhe geführt werden und ferner ein Doppèlboden zum Vorwärmen der Luft vorhanden ist, die durch kleine Locher im Boden in den Innenraum eintritt. (Fig. I.) Von den zehr Größen der Kataloge wïrde ich die mittleren varschlagen, nämlich: Hohe $\times$ Breite $\times$ Tiefe: $28 \times 18 \times 22,45 \times 28 \times 28,40 \times 50 \times 35$, und zwar aus Stahlblech mit zwei Ösen zum Aufhängen, mit Brennerhalter und hoch und niedrig zu stellendem Kronenbrenner. An Stelle der üblichen zwei Tuben a uf der Oberseite, empfehle ich drei anzubringen,
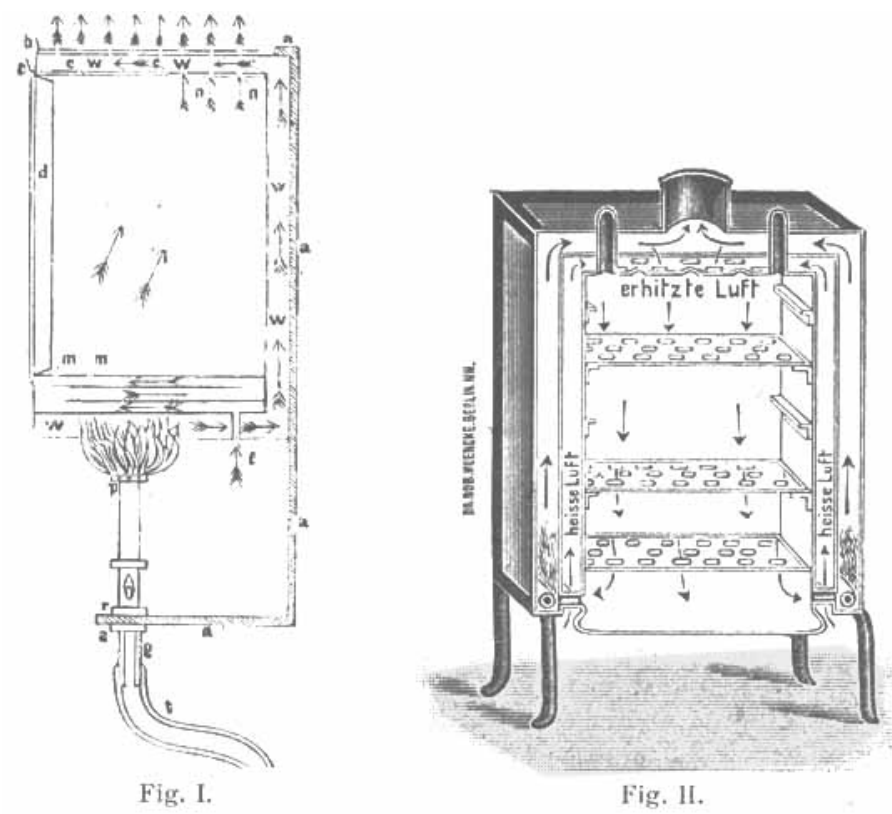

da diese Kästen gern mit Thermoregulator benutzt werden. Die Temperatur zwischen der unteren und oberen Etage einer mittleren Größe differiert um etwa 3-4 Grad. An Stelle eines Lackanstriches, der zwar gut aussieht aber bald abbrennt, empfehle ich das Einreiben mit Graphit und an der Stelle, wo die Flamme den Boden berührt, ein Kupferblech leicht auswechselbar anzubringen. Gut bewährt haben sich und für grőßere Ausführung allein empfehlenswert sind die Kästen, die in den Katalogen fälschlich als solche mit Regenerativheizung verzeichnet sind. (Fig. II.) Die Heizrohren befinden sich hier in den Seiten- und Rückwänden; die vorgewärmte Luft durchstreicht von oben nach unten den Innenraum und wird den Heizkammern zugeführt. Es wird also die obere Etage die heißere sein. Da der Wasserdampf den Kasten nicht durch die Tuben verläßt, sind nur zwei nötig, zumal ein Thermoregulator leider nicht angebracht werden kann; denn die kleinen Heizflămmchen würden, wenn sie als Restflamme funktionieren sollen, verlosichen. Die Außenbekleidung mit Asbest sollte beibehalten werden, da sie den Laboratoriumsraum vor Wärmestrahlung schützt. Ein Vorzug ist es auch, daß die abziehenden Gase sich in einem Rohr vereinigen, das mit dem Schornstein verbunden werden kann. Diese Kästen werden ebenfalls aus Stahlblech hergestellt, und es stehen die größeren auf hohen, fest angenieteten Füßen und sind mit zwei Türen versehen. Ich würde empfehlen, keine durchlochten Bleche als Einlageböden zu verwenden, sondern starkes, gut verzinktes Drahtgewebe, damit die Durchlüftung vollkommen ist.

Folgende drei Größen dürften genügen:

$$
30 \times 25 \times 20, \quad 45 \times 28 \times 28, \quad 45 \times 60 \times 30 .
$$

$\mathrm{Zu}$ erwäbnen sei noch, daß die letzten beiden Modelle außer als Trockenschränke auch für die Sterilisierung von bakteriologischen Geräten durch heiße Luft verwandt werden. Damit ist die Beschreibung der Schränke, bei denen die Luft direkt erhitzt wird, erschöpft, wenn es auch noch zahlreiche andere Konstruktionen mit unwesentlichen Änderungen gibt. Für die Normenblätter empfiehlt es sich, auł alle Einzelheiten genau einzugehen und auch die für den Klempner so wichtigen Durchschnitte zu zeichnen, damit die Herstellung ein heitlich ist.

[A. 207.]

\section{Berichtigung.}

Prof. E. Stiasny ersucht uns unter Bezugnahme auf den Bericht der letzten Hauptversammlung des Internationalen Vereins der Leder-Industrie-Chemiker (Âng. Chem. 33, I, 317 [1920]) daraut hinzuweisen, daß der Vorschlag einer Namensänderung des Vereins (Hinweglassung des Wortes "Internationaler") nicht von ihm ausgegangen sei und auch nicht seine Zustimmung finde. 